\title{
USEFUL BUG BIOLOGY
}

The Biology of Microorganisms. Edited by Arnold L. Demain and Nadine Solomon. Pp. 573. ISBN 0-80532451-8. \$41.95. (Benjamin Cummings, Menlo Park, CA: 1985).

Q iology of Industrial Microorganisms $\mathrm{B}$ is a one volume collection of reviews on the taxonomy, morphology, physiology, ecology, and genetics of a variety of microrganisms. Some are currently useful in industry while others are potentially useful. All of the chapters have been authored by individuals who are experts in the biology of the particular microorganisms discussed. These include Escherichia coli, Pseudomonas, and Xanthomonas species, bacilli, Clostridium species, a variety of glutamic, acetic, lactic, and propionic acid bacteria, methanogens, methylotrophs, $Z y$ monas species, actinomycetes (including Streptomyces species), Penicillium, Cephalosporium, Claviceps, and Trichoderma species, and yeasts (including Saccharomyces species).

The chapters on bacilli (by L. A. Bulla and J. A. Hoch) and Zymomonas species (by B. S. Montenecourt) are particularly useful and informative. These chapters provide well-balanced biological information in a manner that can be readily related to existing molecular biology technology. In addition, there is ample discussion of the industrial utility, both existing and potential. In the chapter on bacilli, there is well organized and informative discussion of sporulation, spore germination, physiology, genetics, and industrial applications of several Bacillus species. The section on genetics includes an update on gene cloning, and the section on industrial application systematically reviews the kinds of useful compounds made by bacilli. The chapter on Zymomonas species begins with an interesting discussion of its potential as a large scale ethanol producer, one of the reasons it is becoming more popular as a research organism. The several sections that follow contain a wealth of detailed biological information on taxonomy, industrial utility, growth, physiology, biochemistry, and genetics. Both this chapter and the chapter on bacilli are excellent sources of information for the scientist with little background in these organisms.

The book has other good sections, too many to detail in this review. Among these are a 15-page discussion of general taxonomy and, in particular, the systematics of fungal taxonomy. Those who are particular about the naming of organisms, and those who should be particular about such things, will find this section very helpful. The chapter on the biology of Streptomyces species is also very good. It generally avoids discussing the molecular biology of streptomycetes that has been repeatedly reviewed and provides a succinct outline of the state of knowledge of this important genus. The chapters on actinomycetes that are not members of the genus Streptomyces, Penicillium species, and Cephalosporium acremonium species are also good sources for background information.

In the Preface, the editors mention that the chapters are unavoidably uneven in treatment. This is because certain aspects of biology (nutrition or genetics, for example) have been more widely studied in some genera than in others. The editors further mention that the unevenness might stimulate scientists to work in areas

\section{CYTOMUT BREEDERS TAKE NOTE}

Practical Flow Cytometry. Howard M. Shapiro. Pp. 295. ISBN 0-8451-02435. \$49.50. (Alan R. Liss, Inc., New York: 1985).

This book is one of several just published or about to be published aimed at the rapidly expanding market of researchers interested in using flow cytometry as a tool in cell and molecular biology. It represents a highly personal view of history, instrumentation, and applications in this field by an author who has participated in development of this technology from its early days and who has chronicled its evolution with considerable wit.

The book opens with an Overture that have been ignored in the past. These areas become apparent upon reading the book but are not as obvious as they could have been, largely because each chapter has been outlined and organized differently. Only two of the 18 chapters have separate sections on industrial utility. Some of the other chapters discussed industrial utility at some point while others did not. Ecology was discussed near the beginning of some chapters, closer to the end of others, and, in some instances, not discussed or discussed as part of another section. Two of the 18 chapters provided a glossary of key terms. This feature was helpful and could have been included for the other 16 .

A knowledge of the biology of a microorganism is essential for successful research, whether fundamental or applied. Because of its content, and because it is unique, this volume will be a worthwhile addition to academic and industrial scientific libraries. It is also a recommended acquisition for individuals who require a desktop source of biological information about a variety of industrial microorganisms.

Jeffrey $T$. Fayerman is a senior scientist at Lilly Research Laboratories, Indianapolis, IN.

defining flow cytometry and its relationship to microscopy and analytical cytology. A brief chapter on Prerequisites provides an excellent summary of pertinent review articles and general references for flow cytometry and related fields with which its users must have at least a nodding acquaintance-electronics, optics, and computers. A detailed history of analytical cytology and How cytometry in Chapter 3 is divided into Classical (pre-Shapiro) and Modern (post-Shapiro) periods and is very well referenced. This is true in the book in general, and the 600-odd references in the back represent what must be a fairly complete compendium of publications in the field up to approxi- 